\title{
Action Experience and Action Discovery in Medicated Individuals with Parkinson's Disease
}

\author{
Jeffery G. Bednark ${ }^{1 *}$, John N. J. Reynolds ${ }^{2}$, Tom Stafford ${ }^{3}$, Peter Redgrave ${ }^{3}$ and \\ Elizabeth A. Franz ${ }^{4 *}$ \\ ${ }_{1}^{1}$ Queensland Brain Institute, The University of Queensland, Brisbane St Lucia, QLD, Australia, ${ }^{2}$ Department of Anatomy, \\ Otago School of Medical Sciences, and The Brain Health Research Centre, University of Otago, Dunedin, New Zealand, \\ ${ }^{3}$ Department of Psychology, University of Sheffield, Sheffield, UK, ${ }^{4}$ Department of Psychology and fMRlotago, University of \\ Otago, Dunedin, New Zealand
}

\section{OPEN ACCESS}

Edited by:

Daniela S. Andres,

ETH Zurich, Switzerland

Reviewed by:

Francesca Garbarini,

University of Turin, Italy

Kristina Aurousseau,

Centre de Recherche de l'Institut

Universitaire de Montréal

(CRIUGM), Canada

*Correspondence:

Jeffery G. Bednark

j.g.bednark@gmail.com

Elizabeth A. Franz

Lfranz@psy.otago.ac.nz

Received: 06 June 2016 Accepted: 09 August 2016 Published: 25 August 2016

Citation:

Bednark JG, Reynolds JNJ, Stafford

T, Redgrave P and Franz EA (2016)

Action Experience and Action

Discovery in Medicated Individuals

with Parkinson's Disease.

Front. Hum. Neurosci. 10:427.

doi: 10.3389/fnhum.2016.00427
Parkinson's disease (PD) is a neurodegenerative disorder that markedly affects voluntary action. While regular dopamine treatment can help restore motor function, dopamine also influences cognitive portions of the action system. Previous studies have demonstrated that dopamine medication boosts action-effect associations, which are crucial for the discovery of new voluntary actions. In the present study, we investigated whether neural processes involved in the discovery of new actions are altered in PD participants on regular dopamine treatment, compared to healthy age-matched controls. We recorded brain electroencephalography (EEG) activity while PD patients and agematched controls performed action discovery (AD) and action control tasks. We found that the novelty P3, a component normally present when there is uncertainty about the occurrence of the sensory effect, was enhanced in PD patients. However, AD was maintained in PD patients, and the novelty P3 demonstrated normal learning-related reductions. Crucially, we found that in PD patients the causal association between an action and its resulting sensory outcome did not modulate the amplitude of the feedback correct-related positivity (fCRP), an EEG component sensitive to the association between an action and its resulting effect. Collectively, these preliminary results suggest that the formation of long-term action-outcome representations may be maintained in PD patients on regular dopamine treatment, but the initial experience of action-effect association may be affected.

Keywords: Parkinson's disease, action discovery, ERPs, agency

\section{INTRODUCTION}

Parkinson's disease (PD) is a neurodegenerative disorder caused by the progressive loss of dopamine neurons (Agid and Blin, 1987), which affects both motor and non-motor function. While dopamine medication (usually in the form of levodopa: L-dopa, a precursor of brain dopamine) is commonly used to restore motor function, exogenous elevation of dopamine has been linked to changes in dopamine-related signaling in the basal ganglia (e.g., Gotham et al., 1988; Cools et al., 2001, 2010; Frank et al., 2004; O'Reilly and Frank, 2006; Moustafa et al., 2008). Individuals with $\mathrm{PD}$ who are $O N$ dopamine medication are shown to have increased sensitivity to reward feedback (Frank et al., 2004), exaggerated working memory updating 
(Moustafa et al., 2008), and enhanced action-outcome binding (Moore et al., 2010).

Crucially, the dopaminergic-system has a significant modulatory role in learning (Reynolds et al., 2001), and likely shapes the discovery of novel action-sensory outcome associations that make up adaptive behavior (Redgrave and Gurney, 2006; Redgrave et al., 2008, 2011; Franz, 2012). Based on the temporal dynamics of dopamine activity, it has been recently proposed that the dopamine-system provides both fast responses to salient events and slow responses that are only executed after the information has been highly processed (Joshua et al., 2009). This allows the dopamine-system to participate in both the monitoring of salient stimuli and the evaluation of information for modifying future behavior. These dynamic signals, however, may be affected in dopamine-medicated individuals with PD leading to changes in the monitoring and evaluation of behaviorally relevant stimuli.

Detecting the occurrence of salient external events and evaluating the cause of salient events is essential for learning and maintaining adaptive behavior (Redgrave and Gurney, 2006; Redgrave et al., 2008). Using event-related potentials (ERPs), derived from electroencephalography (EEG), we have previously shown that the discovery of novel action-outcome associations is dependent on an interaction between salience monitoring and evaluation of novel sensory information with regard to the preceding movement (Bednark et al., 2013). Two key ERPs were demonstrated to play a role: the feedback correct-related positivity (fCRP), and the novelty P3.

The fCRP, along with other reward-related potentials, is commonly associated with the monitoring of salient, positive and reward-related outcomes (Holroyd et al., 2008, 2011). Related to action, previous work has shown that the fCRP response to salient outcomes is enhanced when participants have a sense of control over the salient outcome (Li et al., 2011; Bednark et al., 2013; Bednark and Franz, 2014). Conversely, the novelty P3 is thought to reflect the engagement of evaluative processes necessary for learning when a novel stimulus is encountered (Friedman et al., 2001; Jongsma et al., 2006; Sailer et al., 2010). We have shown that the learning of novel actions directly modulates the novelty P3, with the amplitude of the novelty P3 in response to the behaviorally relevant outcome decreasing as task performance improves and participants acquired a novel action (Bednark et al., 2013). Importantly, both of these brain potentials have been associated with dopamine activity (de Bruijn et al., 2005; Poceta et al., 2006; Polich and Criado, 2006; Potts et al., 2006; Holroyd et al., 2008) and may separately reflect dopaminerelated salience monitoring and evaluative processes.

In the present study, we use these brain potentials to investigate whether the dynamics of the dopamine-system, associated with the discovery of new actions, are altered in individuals with PD while $O N$ regular dopamine treatment (forms of L-dopa), compared to healthy age-matched controls. To test this, medicated individuals with PD and age-matched controls performed action discovery (AD) and action control tasks similar to our previous study (Bednark et al., 2013). Given that dopamine is implicated in mediating both the fCRP and the novelty P3, we expect that both potentials will be enhanced in PD patients compared to controls. However, dopamine-medication boosting of action-effect associations is hypothesized to alter AD.

\section{MATERIALS AND METHODS}

\section{Participants}

Eight patients with mild to moderate PD were recruited from the participant pool of the Action, Brain, and Cognition Laboratory at Otago, a program that works in close association with the Otago Parkinson's Society, Dunedin, New Zealand (see Table $\mathbf{1}$ for patient details). The inclusion criteria were: no known dementia (Mini-Mental State Exam score $\geq 27$ ), no current depressive symptoms (Geriatric

TABLE 1 | Demographic, pathology, and drug details in Parkinson's disease (PD) patients and controls.

\begin{tabular}{|c|c|c|c|c|c|c|c|}
\hline Patient ID & Gender & Age & UPDRS (motor) & Duration of disease & GDS & MMSE & Medication (mg/day) \\
\hline 1 & $\mathrm{~F}$ & 49 & 17 & 8 & 1 & 30 & $\begin{array}{l}\text { Levodopa (150), benserazide (37.5), } \\
\text { orphenadrine (50) }\end{array}$ \\
\hline 2 & $\mathrm{~F}$ & 77 & 5 & 8 & 0 & 26 & $\begin{array}{l}\text { Levodopa (100), benserazide (28.5), } \\
\text { lisuride (0.2), orphenadrine (50) }\end{array}$ \\
\hline 3 & $\mathrm{M}$ & 72 & 8 & 7 & 2 & 30 & $\begin{array}{l}\text { Levodopa (400), carbidopa (100), } \\
\text { benztropine (1) }\end{array}$ \\
\hline 4 & $\mathrm{~F}$ & 62 & 44 & 12 & 1 & 29 & $\begin{array}{l}\text { Levodopa (96), benserazide (24), } \\
\text { ropinirole (8) }\end{array}$ \\
\hline 5 & $\mathrm{~F}$ & 59 & 18 & 3 & 2 & 29 & Levodopa (700), carbidopa (175) \\
\hline 6 & $\mathrm{~F}$ & 66 & 49 & 9 & 2 & 28 & Levodopa (400), carbidopa (100) \\
\hline 7 & $\mathrm{~F}$ & 68 & 11 & 2 & 2 & 28 & $\begin{array}{l}\text { Levodopa (800), carbidopa (200), } \\
\text { ropinirole (4.5) }\end{array}$ \\
\hline 8 & $\mathrm{M}$ & 69 & 42 & 9 & 4 & 29 & $\begin{array}{l}\text { Levodopa (1950), carbidopa (487.5), } \\
\text { entacapone (600) }\end{array}$ \\
\hline
\end{tabular}

\begin{tabular}{|c|c|c|c|c|c|}
\hline PD Mean (SD) & $2 \mathrm{M}$ and $7 \mathrm{~F}$ & $65.25(8.65)$ & $24.25(17.81)$ & 7.25 (3.28) & $1.75(1.17)$ \\
\hline AMC Mean (SD) & $2 \mathrm{M}$ and $7 \mathrm{~F}$ & $64.88(9.25)$ & - & - & $1.13(1.46)$ \\
\hline
\end{tabular}




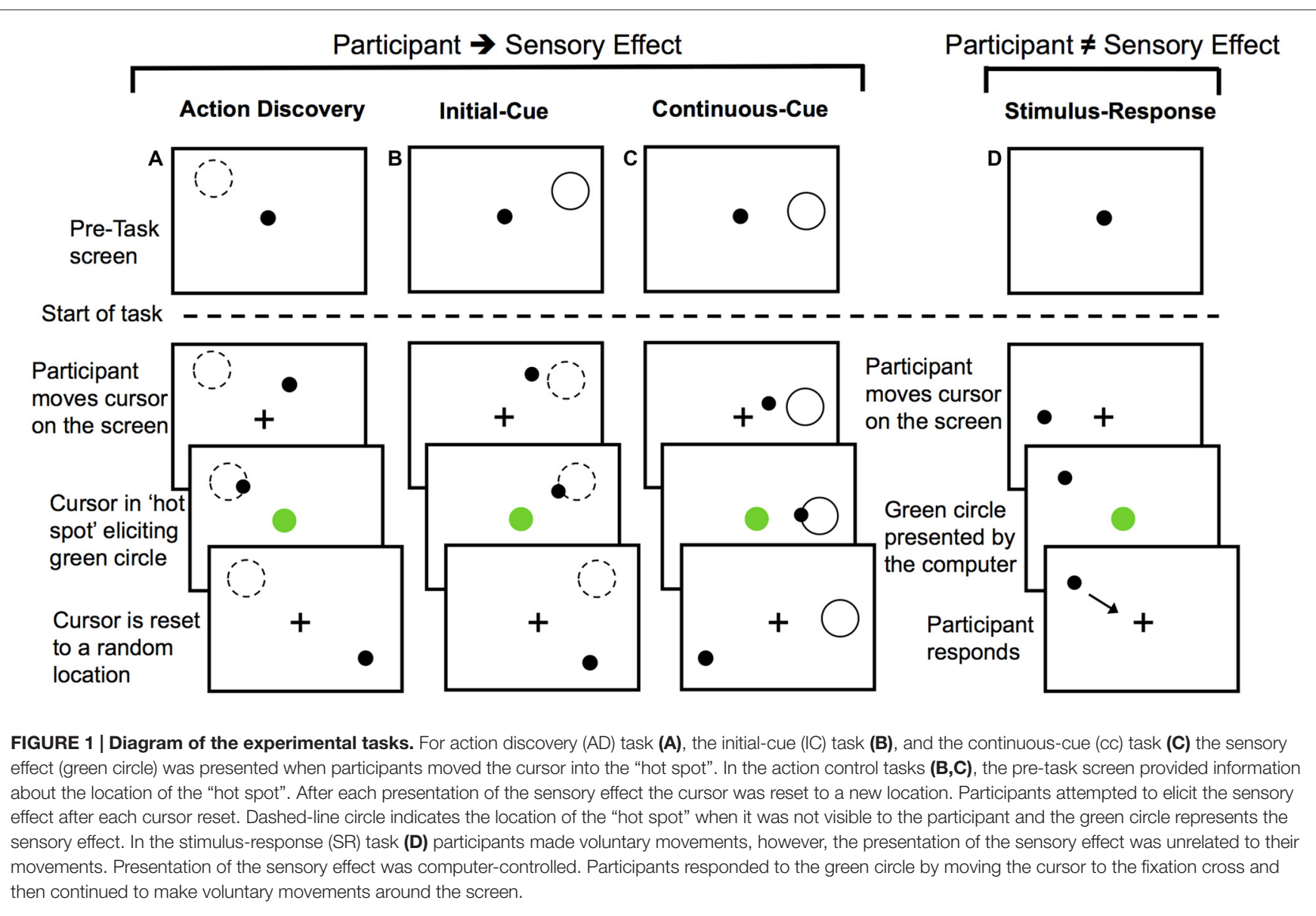

Depression Scale score $\leq 5$ ), and currently on dopamine medication.

Eight age- and sex-matched healthy controls were recruited from a University of Otago database of older adults participants (Table 1). Inclusion criteria were: no known neurological or psychiatric illnesses, no known dementia (MMSE score $\geq 27$ ), and no current depressive symptoms (GDS score $\leq 5$ ). The Lower South Otago Regional Ethics Committee approved all procedures. All participants volunteered and gave their fully informed written consent.

\section{Experimental Procedure}

Prior to the experimental session, patients and controls were interviewed. During the interview, current medical history was obtained, including current PD treatment medication for patients. Neuropsychological tests were also administered to screen for dementia and depression. The motor portion of the UPDRS was administered to patients.

\section{Tasks}

For the $\mathrm{AD}$ and two action control tasks, participants were informed that their goal was to cause a green circle to appear in the center of the screen by moving the cursor on the screen using a tracking-ball mouse. They were further instructed to look at the fixation cross throughout the trials.

For the AD task, participants were not given any specific instructions about how to elicit the green circle, but they were told that they would learn how to do so over the course of the task. A diagram of the AD task can be seen in Figure 1A. The green circle was elicited by the participants' movements when the cursor was moved into a specific location or "hot spot". The location of the "hot spot" remained the same within a block of 30 trials or 30 presentations of the green circle, but each block had a different location for the "hot spot". The cursor was re-located to a new random starting point after each presentation of the green circle. This was done so that the final position, and not the initial position of the movement, was the critical determinant of eliciting the presentation of the green circle. Participants had to then re-locate this location for each trial.

During the $\mathrm{AD}$ task, the monitoring and evaluation of the sensory effect was required to identify the movement(s) eliciting the sensory outcome. Over the course of the AD task, participants learned a set of efficient rules that guided action selection or a movement heuristic. This $\mathrm{AD}$ task was compared to two non-learning goal-directed motor tasks to investigate how the monitoring and evaluation of sensory outcomes may differ 
during $\mathrm{AD}$. As a final control task, we used a stimulus-response (SR) task to investigate the monitoring of behaviorally relevant sensory effects that were unrelated to the preceding voluntary movement.

In the initial cue (IC) action-control task, location of the "hot spot" for eliciting the green circle was cued at the start of the block with a gray outline of a circle (Figure 1B). Participants were instructed that moving the cursor into this screen location would elicit the green circle, but that the gray outline circle would not be present when they performed the task. The cue was then removed and participants began this IC task. This IC provided participants with sensory information for performing the necessary actions for achieving the task goal prior to the start of the task, thereby serving as a control for learning novel actions during the AD task.

The continuous cue (CC) action-control task served as a control for any memory processes or uncertainty (particularly with regards to the novelty $\mathrm{P} 3$ ) associated with remembering the cued location in the IC task or the learnt location in the $\mathrm{AD}$ task. At the start of the $\mathrm{CC}$ task, participants were instructed that moving the cursor into the location defined by a gray outline of a circle on the screen would elicit the green circle (Figure 1C). For action-outcome task (Figures 1A-C) the cursor was re-positioned to a different location on the screen following each presentation of the green circle.

The final control task was a SR task (Figure 1D). For the SR task, participants were informed that their movements did not elicit the sensory effect. Rather, they were instructed to move the cursor around the screen (so participants were still making voluntary movements) and respond to the presentation of the green circle by moving the cursor to the fixation cross (so the green circle was still salient) and then resume moving the cursor around the screen. To control for the reduction in the inter-stimulus interval, which is known to affect P3 amplitude (e.g., Gonsalvez et al., 2007) that is observed with learning in the $\mathrm{AD}$ task, we controlled the timing of green circle presentation. The timing of the green circle in each block of the SR task was computer-controlled to have the same interstimulus interval as the corresponding block of the movementlearning task performed by the same participant (thereby using yoked timing conditions). As a result, any variation or decrease in the timing interval of the sensory effects in the movementlearning task would be directly controlled for by the SR task.

The behavioral tasks that were administered during the experimental session were previously conducted in normal undergraduate participants (Bednark et al., 2013). The theoretical importance of our $\mathrm{AD}$ task has also been highlighted elsewhere (Stafford et al., 2012). There are, however, a few key differences between the tasks used in the present study and those used in our previous ERP study (Bednark et al., 2013). To reduce the difficulty of the tasks, we increased the size of the "hot spot" ( $4.04^{\circ}$ visual angles) and other visual stimuli (i.e., fixation cross: $0.8^{\circ}$; cursor: $0.8^{\circ}$; green sensory effect: $4.04^{\circ}$ ). Additionally, while the three different control tasks were previously conducted in two separate experiments, the same group of participants performed all control tasks in the present study. The order of the tasks was as follows: $\mathrm{ABC}$ ABC-ABC-DDD or ACB-ACB-ACB-DDD. A total of 12 blocks was conducted, with a block defined by 30 presentations of the green circle. The tasks were performed on a $54 \mathrm{~cm}$ display with a black background. MatLab software (MathWorks, Inc.) was used for all stimulus presentation and collection of behavioral responses.

\section{Behavioral Analysis}

For the AD task and the two action control tasks, the time it took the participants to elicit each green circle was recorded. These times were used to determine hit rate, or the number of green circles presented per $2 \mathrm{~s}$ interval. The hit rate was used as a behavioral measure of goaldirected motor performance and was calculated for the first half of green circle occurrences (1-15; F15) and second half of green circle occurrences (16-30; L15) of each block in order to investigate changes in performance within a block.

For the SR task, the time it took the participants to respond to each green circle by moving to the fixation cross, and the number of responses made were recorded. The mean response time and the mean number of responses were computed for the first half of green circle occurrences (1-15; F15) and second half of green circle occurrences (16-30; L15) of each block.

\section{EEG Data Acquisition and Analysis}

EEG and electrooculography (EOG) data were collected continuously using a 32-channel Ag-Ag/Cl sintered Quickcap and a Neuroscan Synamps amplifier, interfaced with a Dell Intel computer running Scan 4.3 software. Data were sampled at 1000 $\mathrm{Hz}$ with a band pass of $0.5-200 \mathrm{~Hz}$, and the gain was $\times 500$. The 28 scalp electrode sites were referenced to linked mastoid electrodes, with $\mathrm{AFz}$ as the ground. Horizontal EOG data were recorded from two electrodes placed on the outer canthi of the two eyes. Vertical EOG data were recorded from linked electrodes on the infraorbital and supraorbital ridges of the left eye. Impedances were maintained below $5 \mathrm{k} \Omega$.

EEG data analysis was conducted offline using purposewritten MatLab scripts. Continuous EEG data were epoched with respect sensory event onset (200 ms prior and $1000 \mathrm{~ms}$ after) in the behavioral task, and baseline corrected relative to the $200 \mathrm{~ms}$ period prior to sensory event onset. Prior to averaging, epochs containing ocular artifacts were corrected (Gratton et al., 1983). To remove movement artifact associated with PD, EEG data were then wavelet decomposed to level 9 using a "Daubechies 6 " discrete wavelet transformation, and reconstructed with 1-25 $\mathrm{Hz}$-frequency range. Based on visual inspection of the averaged waveforms, the mean amplitude of the fCRP for both PD and AMC groups was measured at the $\mathrm{Cz}$ electrode site and averaged across the time window $210-290 \mathrm{~ms}$. For the novelty P3 (measured at $\mathrm{CZ}$ ) we created a mean amplitude measure for the peak amplitude (averaged $25 \mathrm{~ms}$ before and after the peak) found within the 300-450 ms time window. This was done to reduce variation in the latency of the novelty P3 component observed in the patient and control group when the waveforms were visually 
inspected. For plotting purposes, EEG data were smoothed using a one-dimensional digital filter with a $25 \mathrm{~ms}$ time window.

\section{Statistical Analysis}

The hit rate behavioral measure was entered into a mixed analysis of variance (ANOVA) with the within-subjects factors of Task (AD, CC, IC), Block-Half (F15, L15) and Block (Block 1, Block 2, Block 3), and between-subject factor of Group (patients, controls). Similar mixed ANOVAs were conducted for fCRP and novelty P3 amplitude, but the SR task added to the Task factor. To tease apart specific effects of interactions, additional repeated-measures ANOVAs and planned comparisons were used where appropriate to test our hypotheses. Effects were considered significant if $p<0.05$. Greenhouse-Geisser corrections were also applied to $p$-values where appropriate. Effect sizes are shown using partial eta squared $\left(\eta^{2}\right)$. For the assessment of behavioral performance during the SR task, the mean time to response to the stimulus and the mean number of responses to the stimulus were entered into paired-sample $t$-tests that compared the PD and a control group. All statistical tests were conducted using SPSS (version 18.0) software.

\section{RESULTS}

\section{Behavioral Results}

For both PD and controls, the hit rate in each task increased across blocks $\left(\right.$ Block, $F_{(2,28)}=18.78, p<0.001$, partial $\left.\eta^{2}=0.57\right)$ and within blocks (Block-Half, $F_{(1,14)}=37.44, p<0.001$, partial $\left.\eta^{2}=0.73\right)$. There was a significant main effect of Task $\left(F_{(2,28)}=72.48, p<0.001\right.$, partial $\left.\eta^{2}=0.84\right)$ demonstrating that the hit rate during the $\mathrm{AD}$ task $(M=0.85, \mathrm{SE}=0.08)$ was significantly smaller than in the CC task $(M=1.64, \mathrm{SE}=0.09$, $p<0.001)$ and the IC task $(M=1.68, \mathrm{SE}=0.09, p<0.001)$. A significant Task $\times$ Block-Half interaction $\left(F_{(2,28)}=33.59\right.$, $p<0.001$, partial $\left.\eta^{2}=0.71\right)$ suggested that performance was improving at a greater rate during the $\mathrm{AD}$ task. Verifying learning-related improvement, post hoc paired-samples $t$-tests showed that the change in hit rate within a block was significantly larger for the $\mathrm{AD}$ task $(M=0.40, \mathrm{SE}=0.05)$ compared to the CC task $\left(M=0.10, \mathrm{SE}=0.04, t_{(47)}=4.69, p<0.001\right)$ and the IC task $\left(M=-0.06, \mathrm{SE}=0.05, t_{(47)}=8.54\right.$, $p<0.001)$.

Regarding the difference in behavioral performance between groups, we did not find any significant difference between groups in hit rate (Figure 2A). Though the hit rate of the $\mathrm{PD}$ group was generally less than that of the control group. There were also no significant differences between groups in mean response time $\left(F_{(1,14)}=1.69\right.$, $p=0.21$, partial $\left.\eta^{2}=0.11\right)$ and number of responses $\left(F_{(1,14)}=0.19, p=0.67\right.$, partial $\left.\eta^{2}=0.01\right)$ during the SR task (Figures 2B,C).

\section{fCRP Results}

The average ERP waveforms for each task and group are presented in Figure 3. We found a significant Task $\times$ Group

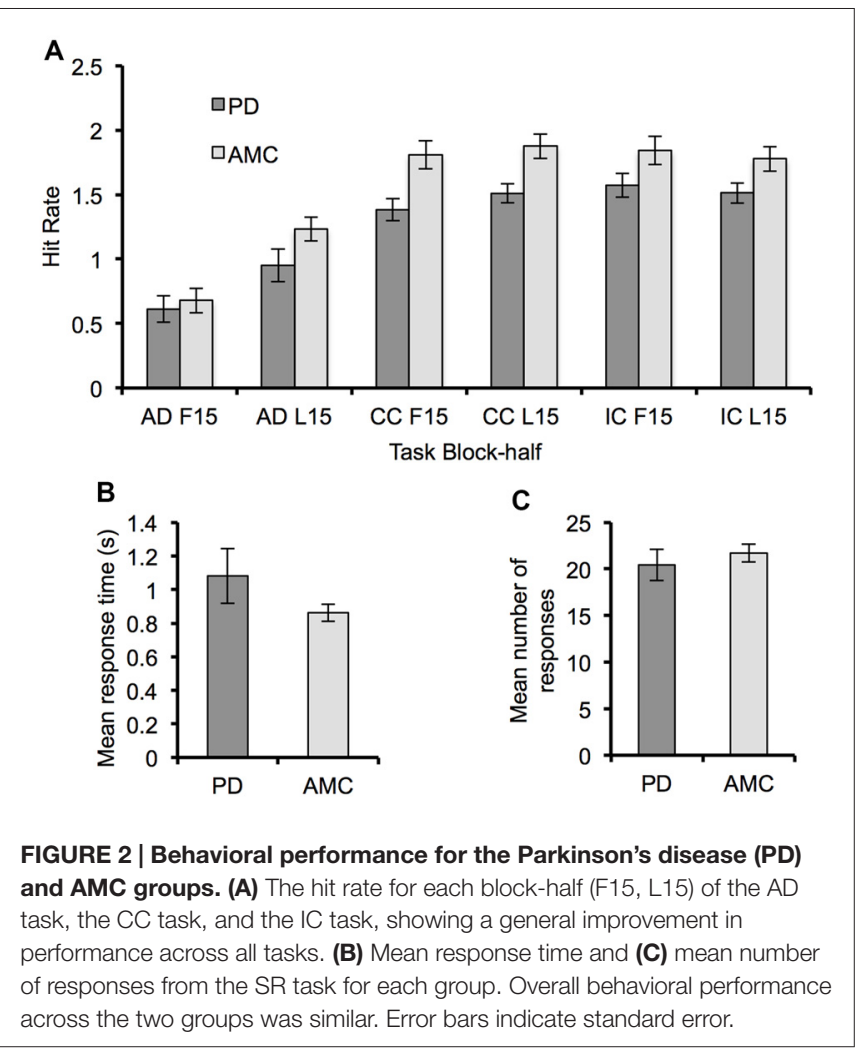

interaction in the amplitude of the $\operatorname{fCRP}\left(F_{(3,42)}=3.73, p=0.03\right.$, partial $\eta^{2}=0.21$ ). As shown in Figure 4A, subsequent post hoc analysis revealed that the magnitude of the fCRP during the SR task was significantly larger in amplitude in the PD group $(M=6.53 \mu \mathrm{V}, \mathrm{SE}=0.80 \mu \mathrm{V})$ compared to the control group $\left(M=3.28 \mu \mathrm{V}, \mathrm{SE}=0.80 \mu \mathrm{V} ;\right.$ Group, $F_{(1,14)}=8.40, p=0.012$, partial $\eta^{2}=0.375$; Bonferroni corrected $\alpha$-level $=0.0125$ ). Initial analysis also suggests that the magnitude of the fCRP response for both groups demonstrated a reduction across Block-Half $\left(F_{(1,14)}=4.84, p=0.045\right.$, partial $\left.\eta^{2}=0.26\right)$ and Block $\left(F_{(2,28)}=5.15, p=0.013\right.$, partial $\left.\eta^{2}=0.27\right)$. However, when each group was assessed individually, only the PD group demonstrated significant reductions in fCRP amplitude across Block-Half $\left(F_{(1,14)}=4.84, p=0.045\right.$, partial $\left.\eta^{2}=0.26\right)$ and Block $\left(F_{(2,28)}=5.15, p=0.013\right.$, partial $\left.\eta^{2}=0.27\right)$. In the control group, there was a significant main-effect of Task $\left(F_{(3,21)}=4.48, p=0.033\right.$, partial $\left.\eta^{2}=0.39\right)$ with the difference observed between the $\mathrm{AD}$ task $(M=5.14 \mu \mathrm{V}$, $\mathrm{SE}=0.60 \mu \mathrm{V})$ and the SR task $(M=3.28 \mu \mathrm{V}, \mathrm{SE}=0.60 \mu \mathrm{V}$, $p=0.022$; Bonferroni corrected $\alpha$-level $=0.016)$, as shown in Figure 4A.

\section{Novelty P3 Results}

As shown in Figure 4B, the magnitude of the novelty P3 response in the PD group was significantly larger than the novelty P3 response in the control group (Group, $F_{(2,14)}=4.48, p=0.034$, partial $\left.\eta^{2}=0.43\right)$. However, while the novelty P3 amplitude for the PD group was larger than in the control group, the general trend of novelty $\mathrm{P} 3$ response is similar for the two 

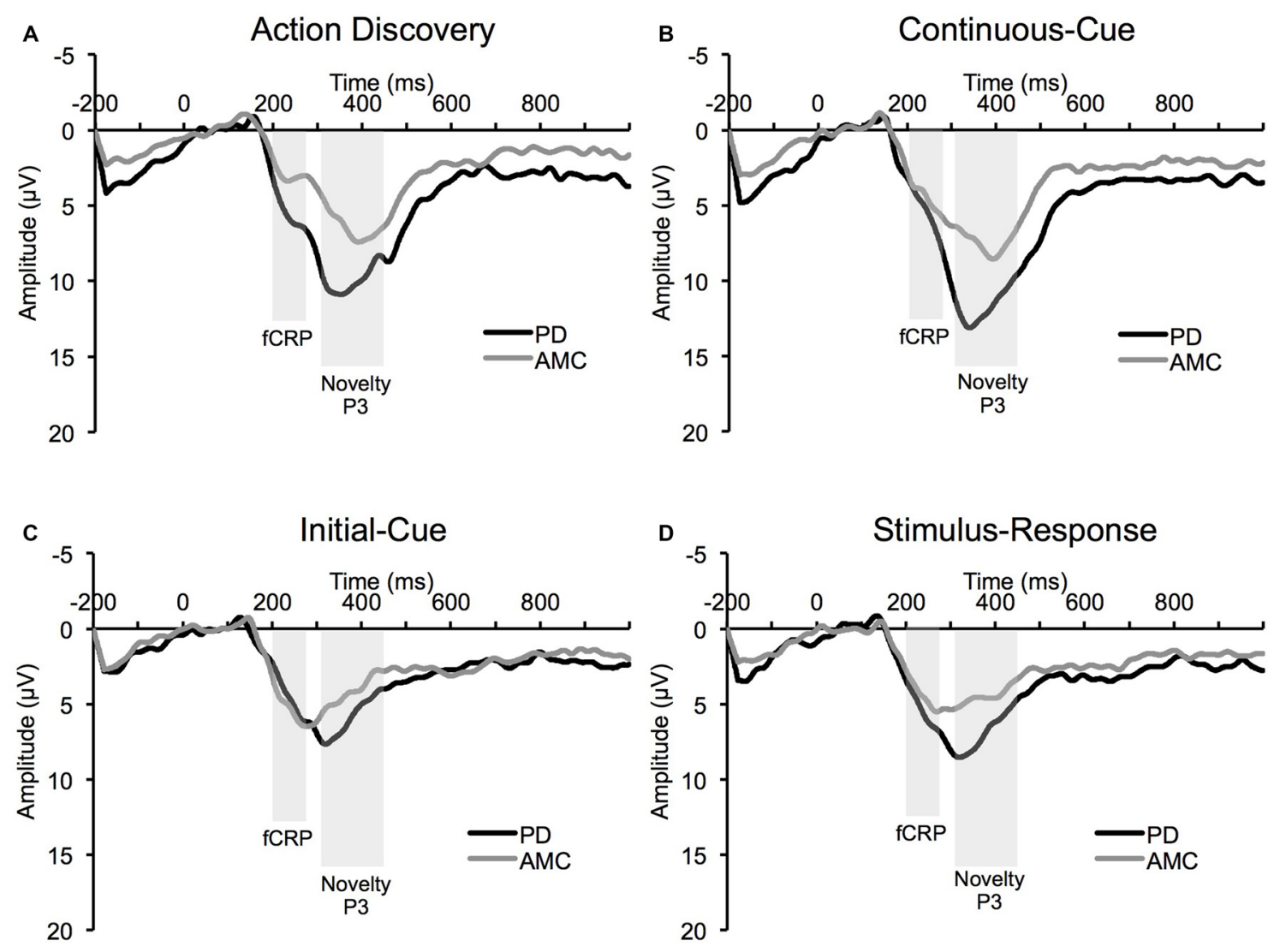

FIGURE 3 | Average event-related potential (ERP) waveform at Cz electrode site from the PD group and the AMC group during (A) the AD task, (B) the CC task, (C) the IC task, and (D) the SR task.

groups (Figure 4C). There was also a significant main-effect of Task overall, $\left(F_{(3,42)}=4.48, p=0.034\right.$, partial $\left.\eta^{2}=0.43\right)$. Bonferroni pair-wise comparisons revealed that the novelty $\mathrm{P} 3$ response during the $\mathrm{AD}$ task $(M=11.77 \mu \mathrm{V}, \mathrm{SE}=0.89 \mu \mathrm{V})$ was significantly larger than in the IC task $(M=7.03 \mu \mathrm{V}$, $\mathrm{SE}=0.49 \mu \mathrm{V}, p<0.001)$ and $\mathrm{CC}$ task $(M=7.53 \mu \mathrm{V}, \mathrm{SE}=0.48$ $\mu \mathrm{V}, p<0.001)$. The SR task $(M=10.65 \mu \mathrm{V}, \mathrm{SE}=0.96 \mu \mathrm{V})$ was also significantly different from the IC task $(p=0.003)$ and the CC task $(p=0.017)$. However, no difference between the AD task and the SR task was observed. The novelty P3 response also demonstrated a significant reduction across BlockHalf $\left(F_{(1,14)}=4.48, p=0.034\right.$, partial $\left.\eta^{2}=0.43\right)$, and Block $\left(F_{(2,28)}=4.48, p=0.034\right.$, partial $\left.\eta^{2}=0.43\right)$.

This pattern of significant main effects was observed in both PD and control groups, as confirmed by separate analyses on each group. Accordingly, in the PD group, there were significant main effects of Task $\left(F_{(3,21)}=14.13, p=0.001\right.$, partial $\left.\eta^{2}=0.67\right)$, Block-Half $\left(F_{(1,7)}=27.27, p=0.001\right.$, partial $\left.\eta^{2}=0.80\right)$, and Block $\left(F_{(2,12)}=6.04, p=0.021\right.$, partial $\left.\eta^{2}=0.46\right)$. The pattern was similar for the control group: Task $\left(F_{(3,21)}=7.36, p=0.006\right.$, partial $\left.\eta^{2}=0.51\right)$, Block-Half $\left(F_{(1,7)}=13.59, p=0.008\right.$, partial $\left.\eta^{2}=0.66\right)$, and Block $\left(F_{(2,14)}=6.30, p=0.027\right.$, partial $\left.\eta^{2}=0.47\right)$.

\section{DISCUSSION}

The present study investigated the neural processes mediating the discovery of novel actions in PD patients currently receiving dopamine treatment. We found no significant behavioral differences in $\mathrm{AD}$ between $\mathrm{PD}$ patients and age-matched controls. However, ERP analysis suggests that there may be significant alterations to the neural processes involved in the formation of action-outcome associations in PD patients compared to controls.

\section{Novelty P3}

As hypothesized, the amplitude of the novelty P3 was larger in people with PD compared to age-matched controls. This effect is complementary to previous work that has demonstrated a reduction in novelty P3 amplitude in PD patients OFF dopamine medication (Poceta et al., 2006). Indeed, evidence from multiple studies suggests that dopamine is a crucial neuromodulator of the novelty P3 response (Polich and Criado, 2006; Polich, 2007). This would suggest that dopamine levels modulate the response to salient unexpected sensory events, which is in keeping with the dopamine errorsignaling hypothesis (Schultz, 1997, 1998). Additionally, this 
fCRP

A

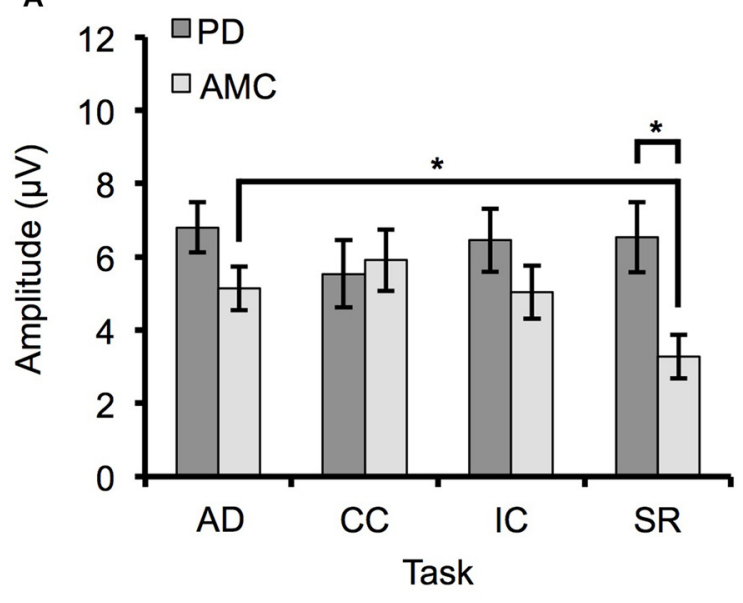

C

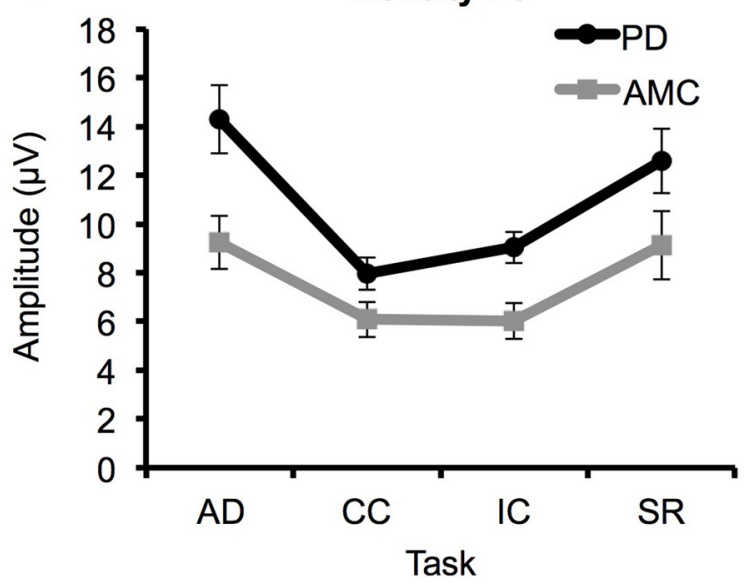

B

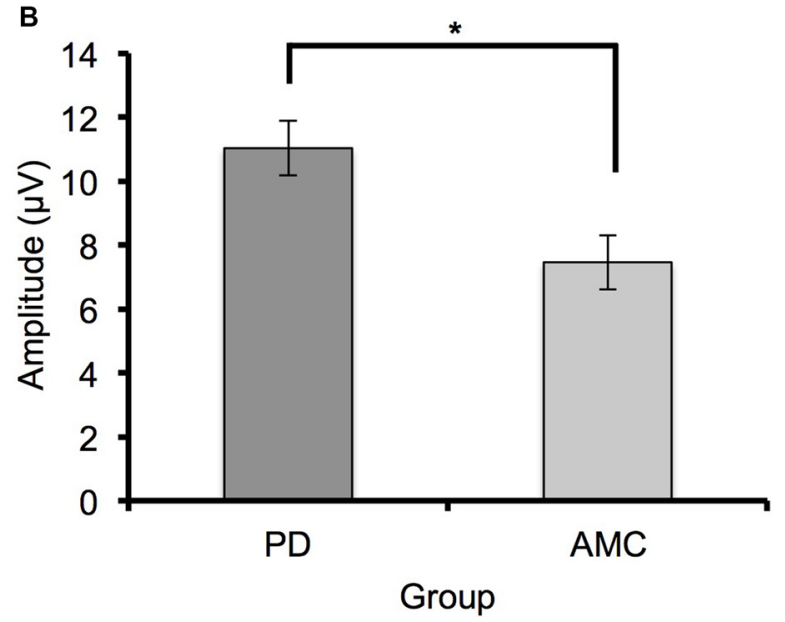

FIGURE 4 | ERP results for PD and AMC groups. (A) Feedback correct-related positivity (fCRP) amplitude in the PD and AMC groups for the AD task, CC task, IC task, and SR task. There was a significant difference in fCRP amplitude between the PD group and the AMC group in SR. For the AMC group, fCRP amplitude in SR was significantly reduced compared to AD. (B) There was an overall significant enhancement in novelty P3 amplitude in the PD group compared to the AMC group. (C) However, the general trend of the novelty P3 was similar across the different tasks for both groups. Error bars indicate standard error. * $p<0.05$.

enhancement of the novelty P3 provides further evidence for the proposed over-abundance of dopamine in relatively preserved portions of the basal ganglia (Gotham et al., 1988; Swainson et al., 2000; Cools et al., 2001; Shohamy et al., 2006). In contrast to previous EEG studies, our results suggest that the mechanisms governing the reduction of the novelty P3 are unaffected or restored in medicated individuals with PD.

In both PD patients and age-matched controls, the novelty P3 demonstrated learning-related reductions within and across blocks of the AD task. Previously, we hypothesized that the novelty $\mathrm{P} 3$ reflects the engagement of attentional mechanisms necessary for identifying the movements responsible for the unexpected sensory event (Franz, 2012; Bednark et al., 2013). As the responsible movements are identified and a heuristic is formed to guide future action selection, the need to engage attentional mechanisms is reduced. In the present study, we found that the novelty $\mathrm{P} 3$ response in $\mathrm{PD}$ patients still demonstrated learning-related reductions. This would suggest that neural processes mediating action-outcome anticipation might be unaffected by changes to the magnitude of the dopamine response or that dopamine medication restores function to this system.

According to previous $\mathrm{AD}$ proposals of the dopamine error signal, the formation of long-term associations between actions and outcomes are likely established in structures outside the basal ganglia (Redgrave and Gurney, 2006; Redgrave et al., 2008). One such brain structure is anterior cingulate cortex (ACC). Recent evidence indicates that the ACC is responsible for governing the value of actions based on their previous reinforcement history (Walton et al., 2004; Rushworth et al., 2007; Holroyd and Coles, 2008). The ACC is the proposed 
source generator of the novelty P3 (Polich, 2007), and is thought to receive the dopamine error signal from the basal ganglia (Holroyd and Coles, 2002, 2008). While original proposals of this ACC-dopamine error signaling (Holroyd and Coles, 2002) have focused on prediction errors that are derived from subcortical indications of stimulus novelty (for a review see Redgrave et al., 2008, 2011), recent evidence from animal studies suggests that the dopamine error signal (with a longer latency) can be elicited from cortical indications of stimulus novelty (Bromberg-Martin et al., 2010; Nomoto et al., 2010). Along these lines, there is evidence that the ACC can directly modulate dopamine release (Gariano and Groves, 1988). Indeed, it has been proposed that different time scales of the dopamine response may control different aspects of motor behavior (Joshua et al., 2009). Thus, the ACC may govern the learningrelated reductions in the novelty $\mathrm{P} 3$ response through its modulation of dopamine signaling. This possible top-down mechanism appears to remain moderately intact or is restored to normal function in $\mathrm{PD}$ patients on regular dopamine treatment.

\section{fCRP}

In the motor tasks in which participants' actions were responsible for the occurrence of the sensory event, there was no significant difference between PD patients and agematched controls. Crucially, when the participants' actions were unrelated to the occurrence of the sensory event, we observed an enhancement in the fCRP response in PD patients compared to age-matched controls. As highlighted in the Introduction, previous studies have demonstrated that the fCRP response is enhanced by the perceived responsibility over $\mathrm{Li}$ et al. (2011) and coupling of an intentional action with a sensory event (Bednark et al., 2013; Bednark and Franz, 2014). Additionally, it has been previously demonstrated that PD patients on regular dopamine treatment perceive actions and sensory events as occurring closer in time than when OFF dopamine treatment (Moore et al., 2010). These converging lines of evidence would suggest that PD patients had an exaggerated experience of association between their actions and the sensory effect during the SR task. This is despite explicit knowledge that their actions were not responsible for the sensory effect. Presumably this could be because dopamine treatment used to restore dopamine levels in $\mathrm{PD}$ patients may actually reduce the dynamic range of the dopamine response (Frank, 2005). This, in turn, may reduce the fCRP differentiation between action-related and action-unrelated sensory effects.

In contrast to the novelty $\mathrm{P} 3$, the pattern of fCRP response across and within tasks in PD patients varied significantly from the fCRP pattern observed in age-matched controls. This would suggest that the processes involved in the fCRP response might be altered in PD patients. A recent source localization study that used principal component analysis to remove the influence of other ERPs demonstrated that the basal ganglia is the likely source generator of the reward-related fCRP response (Foti et al., 2011). Applied to our findings, this would suggest that alterations to the fCRP response in PD patients may occur at the level of the basal ganglia. Thus, in PD patients $O N$ medication, it appears that over-abundant dopamine levels in relatively preserved portions of the basal ganglia causes the behavioral relevance of the sensory effect to be maintained across all tasks.

An alternative possibility is that the enhanced fCRP may be related to increased task motivation. Others have suggested that task motivation can modulate brain activity in the time-range of the fCRP (Hajcak et al., 2005; Boksem et al., 2006; Sailer et al., 2010). However, it is unlikely that task motivation in the PD patients was only enhanced during the SR task. Nevertheless, reduced task motivation may explain the observed reduction in fCRP amplitude within and across blocks in the PD patients.

\section{Limitations and Future Directions}

The low number of individuals with PD available for this study and the lack of an OFF-medication state in the PD group limits the extent to which these results can be generalized; the present findings must be viewed as preliminary. However, it is important to note that despite the small sample size and heterogeneity of the PD observed in these patients, we were still able to find significant differences in our ERP measures. Thus, this study provides initial evidence for the use of ERPs in exploring PD. Future studies with access to larger patient populations and ON/OFF design should be conducted to determine the extent to which these ERPs are influenced by dopamine medication during agent-based action learning. However, performing a study with ON/OFF design may prove to be difficult given the learning nature of this task. Differences between the $O N$ and OFF state may not be detected because carry-over effects (e.g., learning the task) affect performance in the task from one state to the next. Conducting this experiment with de novo PD patients who still have a normal level of motor control could potentially reveal if action learning mechanisms are affected before gross motor control effects are visible.

\section{Conclusion}

This is an initial study investigating whether the ability to identify new actions might be affected in dopamine medicated $\mathrm{PD}$ participants. $\mathrm{AD}$ is maintained in $\mathrm{PD}$ patients on regular dopamine treatment despite a potential over-abundance of dopamine in relatively preserved portions of the basal ganglia. This highlights the importance of structures outside the basal ganglia for the formation of long-term action-outcome representations. However, the initial experience of actionoutcome association appears to be affected by increased dopamine levels in the basal ganglia.

\section{AUTHOR CONTRIBUTIONS}

JGB: designed and conducted study, analyzed data, and wrote the manuscript. JNJR: contributed to study design and writing of the manuscript. TS: contributed to study design, the development of the experimental task, and editing the manuscript. PR: contributed to task design and experimental concept; contributed to the editing of the manuscript. EAF: provided the facilities for 
conducting the study; contributed to study design, data analysis, and writing of the manuscript.

\section{ACKNOWLEDGMENTS}

This work was supported in part by a grant from the Parkinson's Society of New Zealand (to EAF) and in part by a Marsden grant

\section{REFERENCES}

Agid, Y., and Blin, J. (1987). Nerve-cell death in degenerative diseases of the central-nervous-system: clinical aspects. Ciba Found. Symp. 126, 3-29.

Bednark, J. G., and Franz, E. A. (2014). Agency attribution: event-related potentials and outcome monitoring. Exp. Brain Res. 232, 1117-1126. doi: 10.1007/s00221014-3821-4

Bednark, J. G., Reynolds, J. N. J., Stafford, T., Redgrave, P., and Franz, E. A. (2013). Creating a movement heuristic for voluntary action: electrophysiological correlates of movement-outcome learning. Cortex 49, 771-780. doi: 10.1016/j. cortex.2011.12.005

Boksem, M. A., Tops, M., Wester, A. E., Meijman, T. F., and Lorist, M. M. (2006). Error-related ERP components and individual differences in punishment and reward sensitivity. Brain Res. 1101, 92-101. doi: 10.1016/j.brainres.2006. 05.004

Bromberg-Martin, E. S., Matsumoto, M., and Hikosaka, O. (2010). Dopamine in motivational control: rewarding, aversive and alerting. Neuron 68, 815-834. doi: 10.1016/j.neuron.2010.11.022

Cools, R., Barker, R. A., Sahakian, B. J., and Robbins, T. W. (2001). Enhanced or impaired cognitive function in Parkinson's disease as a function of dopaminergic medication and task demands. Cereb. Cortex 11, 1136-1143. doi: 10.1093/cercor/11.12.1136

Cools, R., Rogers, R., Barker, R. A., and Robbins, T. W. (2010). Top-down attentional control in Parkinson's disease: salient considerations. J. Cogn. Neurosci. 22, 848-859. doi: 10.1162/jocn.2009.21227

de Bruijn, E. R. A., Hulstijn, W., Verkes, R. J., Ruigt, G. S. F., and Sabbe, B. G. C. (2005). Altered response evaluation-monitoring of late responses after administration of D-amphetamine. J. Psychophysiol. 19, 311-318. doi: 10. 1027/0269-8803.19.4.311

Foti, D., Weinberg, A., Dien, J., and Hajcak, G. (2011). Event related potential activity in the basal ganglia differentiates rewards from nonrewards: temporospatial principal components analysis and source localization of the feedback negativity. Hum. Brain Mapp. 32, 2207-2216. doi: 10.1002/hbm. 21182

Frank, M. J. (2005). Dynamic dopamine modulation in the basal ganglia: a neurocomputational account of cognitive deficits in medicated and nonmedicated Parkinsonism. J. Cogn. Neurosci. 17, 51-72. doi: 10. 1162/0898929052880093

Frank, M. J., Seeberger, L. C., and O'reilly, R. C. (2004). By carrot or by stick: cognitive reinforcement learning in Parkinsonism. Science 306, 1940-1943. doi: 10.1126/science.1102941

Franz, E.A. (2012). The allocation of attention to learning of goal-directed actions: a cognitive neuroscience framework focusing on the basal ganglia. Front. Psychol. 3:535. doi: 10.3389/fpsyg.2012.00535

Friedman, D., Cycowicz, Y. M., and Gaeta, H. (2001). The novelty P3: an eventrelated brain potential (ERP) sign of the brain's evaluation of novelty. Neurosci. Biobehav. Rev. 25, 355-373. doi: 10.1016/s0149-7634(01)00019-7

Gariano, R., and Groves, P. (1988). Burst firing induced in midbrain dopamine neurons by stimulation of the medial prefrontal and anterior cingulate cortices. Brain Res. 462, 194-198. doi: 10.1016/0006-8993(88)90606-3

Gonsalvez, C. J., Barry, R. J., Rushby, J. A., and Polich, J. (2007). Target-totarget interval, intensity, and P300 from an auditory single-stimulus task. Psychophysiology 44, 245-250. doi: 10.1111/j.1469-8986.2007.00495.x

Gotham, A. M., Brown, R. G., and Marsden, C. D. (1988). 'Frontal' cognitive function in patients with Parkinson's disease 'on' and 'off levodopa. Brain 111, 299-321. doi: 10.1093/brain/111.2.299 from the Royal Society of New Zealand (to JNJR), a European Community 7th Framework Programme (FP7/2007-2013, grant agreement No. ICT-IP-231722, to TS and PR) and a Wellcome Trust grant 091409 (to PR). During the preparation of this article, JNJR received support from a Rutherford Discovery Fellowship from the Royal Society of New Zealand, and EAF also received funding from her Marsden grant from the Royal Society of New Zealand.

Gratton, G., Coles, M. G. H., and Donchin, E. (1983). A new method for off-line removal of ocular artifact. Electroencephalogr. Clin. Neurophysiol. 55, 468-484. doi: 10.1016/0013-4694(83)90135-9

Hajcak, G., Moser, J. S., Yeung, N., and Simons, R. F. (2005). On the ERN and the significance of errors. Psychophysiology 42,151-160. doi: 10.1111/j.1469-8986. 2005.00270.x

Holroyd, C. B., and Coles, M. G. H. (2002). The neural basis of human error processing: reinforcement learning, dopamine and the errorrelated negativity. Psychol. Rev. 109, 679-709. doi: 10.1037/0033-295x.109. 4.679

Holroyd, C. B., and Coles, M. G. H. (2008). Dorsal anterior cingulate cortex integrates reinforcement history to guide voluntary behavior. Cortex 44, 548-559. doi: 10.1016/j.cortex.2007.08.013

Holroyd, C. B., Krigolson, O. E., and Lee, S. (2011). Reward positivity elicited by predictive cues. Neuroreport 22, 249-252. doi: 10.1097/wnr.0b013e328345441d

Holroyd, C. B., Pakzad-Vaezi, K. L., and Krigolson, O. E. (2008). The feedback correct-related positivity: sensitivity of the event-related brain potential to unexpected positive feedback. Psychophysiology 45, 688-697. doi: 10.1111/j. 1469-8986.2008.00668.x

Jongsma, M. L. A., Eichele, T., Van Rijn, C. M., Coenen, A. M. L., Hugdahl, K., Nordby, H., et al. (2006). Tracking pattern learning with single-trial eventrelated potentials. Clin. Neurophysiol. 117, 1957-1973. doi: 10.1016/j.clinph. 2006.05.012

Joshua, M., Adler, A., and Bergman, H. (2009). The dynamics of dopamine in control of motor behavior. Curr. Opin. Neurobiol. 19, 615-620. doi: 10.1016/j. conb.2009.10.001

Li, P., Han, C., Lei, Y., Holroyd, C. B., and Li, H. (2011). Responsibility modulates neural mechanisms of outcome processing: an ERP study. Psychophysiology 48, 1129-1133. doi: 10.1111/j.1469-8986.2011.01182.x

Moore, J. W., Schneider, S. A., Schwingenschuh, P., Moretto, G., Bhatia, K. P., and Haggard, P. (2010). Dopaminergic medication boosts action-effect binding in Parkinson's disease. Neuropsychologia 48, 1125-1132. doi: 10.1016/j. neuropsychologia.2009.12.014

Moustafa, A. A., Sherman, S. J., and Frank, M. J. (2008). A dopaminergic basis for working memory, learning and attentional shifting in Parkinsonism. Neuropsychologia 46, 3144-3156. doi: 10.1016/j.neuropsychologia.2008. 07.011

Nomoto, K., Schultz, W., Watanabe, T., and Sakagami, M. (2010). Temporally extended dopamine responses to perceptually demanding reward-predictive stimuli. J. Neurosci. 30, 10692-10702. doi: 10.1523/jneurosci.4828-09.2010

O’Reilly, R. C., and Frank, M. J. (2006). Making working memory work: a computational model of learning in the prefrontal cortex and basal ganglia. Neural Comput. 18, 283-328. doi: 10.1162/089976606775093909

Poceta, S. J., Houser, M., and Polich, J. (2006). Event-related potentials in restless legs syndrome and Parkinson's Disease (abstract). Sleep 28, A274.

Polich, J. (2007). Updating P300: an integrative theory of P3a and P3b. Clin. Neurophysiol. 118, 2128-2148. doi: 10.1016/j.clinph.2007.04.019

Polich, J., and Criado, J. R. (2006). Neuropsychology and neuropharmacology of P3a and P3b. Int. J. Psychophysiol. 60, 172-185. doi: 10.1016/j.ijpsycho.2005. 12.012

Potts, G. F., Martin, L. E., Burton, P., and Montague, P. R. (2006). When things are better or worse than expected: the medial frontal cortex and the allocation of processing resources. J. Cogn. Neurosci. 18, 1112-1119. doi: 10.1162/jocn.2006. 18.7.1112

Redgrave, P., and Gurney, K. (2006). The short-latency dopamine signal: a role in discovering novel actions? Nat. Rev. Neurosci. 7, 967-975. doi: 10.1038/nrn2022 
Redgrave, P., Gurney, K., and Reynolds, J. (2008). What is reinforced by phasic dopamine signals? Brain Res. Rev. 58, 322-339. doi: 10.1016/j.brainresrev.2007. 10.007

Redgrave, P., Vautrelle, N., and Reynolds, J. N. J. (2011). Functional properties of the basal ganglia's re-enterant loop architecture: Selection and reinforcement. Neuroscience 198, 138-151. doi: 10.1016/j.neuroscience.2011. 07.060

Reynolds, J. N. J., Hyland, B. I., and Wickens, J. R. (2001). A cellular mechanism of reward-related learning. Nature 413, 67-70. doi: 10.1038/35092560

Rushworth, M. F. S., Behrens, T. E. J., Rudebeck, P. H., and Walton, M. E. (2007). Contrasting roles for cingulate and orbitofrontal cortex in decisions and social behaviour. Trends Cogn. Sci. 11, 168-176. doi: 10.1016/j.tics.2007. 01.004

Sailer, U., Fischmeister, F. P., and Bauer, H. (2010). Effects of learning on feedbackrelated brain potentials in a decision-making task. Brain Res. 1342, 85-93. doi: 10.1016/j.brainres.2010.04.051

Schultz, W. (1997). Dopamine neurons and their role in reward mechanisms. Curr. Opin. Neurobiol. 7, 191-197. doi: 10.1016/s0959-4388(97)80007-4

Schultz, W. (1998). Predictive reward signal of dopamine neurons. J. Neurophysiol. $80,1-27$.

Shohamy, D., Myers, C. E., Geghman, K. D., Sage, J., and Gluck, M. A. (2006). L-dopa impairs learning, but spares generalization, in Parkinson's disease. Neuropsychologia 44, 774-784. doi: 10.1016/j.neuropsychologia.2005.07.013
Stafford, T., Thirkettle, M., Walton, T., Vautrelle, N., Hetherington, L., Port, M., et al. (2012). A novel task for the investigation of action acquisition. PLoS One 7:e37749. doi: 10.1371/journal.pone.0037749

Swainson, R., Rogers, R. D., Sahakian, B. J., Summers, B. A., Polkey, C. E., and Robbins, T. W. (2000). Probabilistic learning and reversal deficits in patients with Parkinson's disease or frontal or temporal lobe lesions: possible adverse effects of dopaminergic medication. Neuropsychologia 38, 596-612. doi: 10 1016/s0028-3932(99)00103-7

Walton, M. E., Devlin, J. T., and Rushworth, M. F. S. (2004). Interactions between decision making and performance monitoring within prefrontal cortex. Nat. Neurosci. 7, 1259-1265. doi: 10.1038/nn1339

Conflict of Interest Statement: The authors declare that the research was conducted in the absence of any commercial or financial relationships that could be construed as a potential conflict of interest.

Copyright (c) 2016 Bednark, Reynolds, Stafford, Redgrave and Franz. This is an openaccess article distributed under the terms of the Creative Commons Attribution License (CC BY). The use, distribution and reproduction in other forums is permitted, provided the original author(s) or licensor are credited and that the original publication in this journal is cited, in accordance with accepted academic practice. No use, distribution or reproduction is permitted which does not comply with these terms. 